\title{
Dense Nanoparticles Exhibit Enhanced Vascular Wall Targeting over Neutrally Buoyant Nanoparticles in Human Blood Flow
}

\section{Alex J. Thompson and Omolola Eniola-Adefeso*}

\author{
A.J.Thompson ${ }^{1}$ \\ *Corresponding Author: \\ Prof. O. Eniola-Adefeso ${ }^{1,2}$ \\ E-mail: lolaa@umich.edu
}

${ }^{1}$ Department of Chemical Engineering, ${ }^{2}$ Department of Biomedical Engineering, University of Michigan, Ann Arbor, MI 48109.

\begin{abstract}
For vascular-targeting carrier (VTC) systems to be effective, carriers must be able to localize and adhere to the vascular wall at the target site. Research suggests that neutrally buoyant nanoparticles are limited by their inability to localize to the endothelium, making them suboptimal as carriers. This study examines whether particle density can be exploited to improve the targeting (localization and adhesion) efficiency of nanospheres to the vasculature. Silica spheres with $500 \mathrm{~nm}$ diameter, which have a density roughly twice that of blood, exhibit improved adhesion to inflamed endothelium in an in vitro model of human vasculature compared to neutrally buoyant polystyrene spheres of the same size. Silica spheres also display better near-wall localization in the presence of red blood cells than they do in pure buffer, likely resulting in the observed improvement in adhesion. Titania spheres (4 times more dense than blood) adhere at levels higher than polystyrene, but only in conditions when gravity or centrifugal force acts in the direction of adhesion. In light of the wide array of materials proposed for use as carrier systems for drug delivery and diagnostics, particle density may be a useful tool for improving the targeting of diseased tissues.
\end{abstract}

Keywords: drug delivery, hemodynamics, inflammation, nanoparticles, vascular targeting 


\section{Introduction}

Improvements in the design of injectable carrier systems that actively target diseased tissues via the endothelium could potentially improve the diagnosis and treatment of a wide variety of human diseases, including cancer and atherosclerosis. The ability of the carrier to localize (or marginate) to the blood vessel wall is an important requisite for successful targeting to the endothelium. It has been established that particle size and geometry significantly affects the ability of a particle to marginate and adhere to the vascular wall in human blood flow.[1-7] However, it is quite possible that other particle characteristics, in addition to size, may affect the margination propensity of a carrier system. One such parameter is particle density, which can differ depending on the material makeup of the carrier system.

To date, materials with a range of densities have been proposed for use in drug delivery or diagnostics, including microbubbles[8,9], polymers[10-13], liposomes[14,15], inorganic particles (titania, gold, iron oxide)[16-18], blood cells[19,20], or combinations of multiple material types[16,21-29]. For instance, gas-filled microbubbles can have densities roughly $1 \%$ that of blood.[30] Liposomes are often neutrally buoyant, but can be fabricated with varied densities depending on the density of the medium encapsulated within the liposome.[31,32] Some common polymer-based particles which are biocompatible and FDA approved include poly(lactic acid), poly(glycolic acid), poly(methyl methacrylate), polycaprolactone, and PLGA. These polymeric particles tend to have densities either neutrally buoyant or slightly higher than blood.[33] Metal oxides, including silica and titania particles (2.0 and $3.9 \mathrm{~g} \mathrm{~mL}^{-1}$, respectively, in this study) also have been proposed as potential carriers.[33-35] Particle types with even higher densities have been proposed for use as carriers as well, including iron oxide $\left(\sim 5 \mathrm{~g} \mathrm{~mL}^{-1}\right)$ and gold $\left(\sim 19 \mathrm{~g} \mathrm{~mL}^{-1}\right)$ nanoparticles. Also, combinations of different materials have been investigated which may have densities that fall 
between the densities of the individual materials. Such systems include liposomes or solid particles loaded with heavier materials such as gadolinium, magnetite, or gold; which provides benefits for imaging or hyperthermia therapy, for instance. [16,24-29] Despite the wide range of the VTC densities available, there is limited understanding of the role that density plays in prescribing carrier performance.

In order for a targeted delivery system to be effective, it should be able to marginate to the vascular wall effectively in blood flow. It is widely known that red blood cells (RBC) play a crucial role in the margination of leukocytes and platelets. By congregating in the center of flow, RBCs force white cells and platelets to concentrate in the "cell-free layer" (CFL) adjacent to the endothelium.[36] This effect aids in the margination and interaction of white cells and platelets with the vascular wall, and is in part due to the size of platelets $(\approx 2-3 \mu \mathrm{m}$ diameter) and white cells (8-12 $\mu \mathrm{m}$ diameter).[37] Studies utilizing in vitro models of human vasculature have shown that neutrally-buoyant microspheres of sufficient size $(\geq 2 \mu \mathrm{m}$ diameter) also can take advantage of this phenomenon.[1-5,38,39] However, previous experimental and computational works have reported that neutrally-buoyant particles with diameters in the $100-500 \mathrm{~nm}$ size range are not preferentially distributed to the vessel wall via this $\mathrm{RBC}$ effect. This inefficient margination results in sub-optimal targeting to endothelium in the presence of physiological levels of RBCs.[3,5,38,39] Still, particles in this size range are attractive as VTCs in that they are able to carry a larger payload than ultrasmall nanoparticles while still being able to safely navigate microvasculature.[40-47] To date, it is unclear whether particle density affects the margination of nanoparticles or sub-micron sized particles to the CFL via interactions with RBCs in blood flow. While a recent study shows that increasing particle density negatively affects nanoparticle margination in a microchannel, this work was done in the absence of blood components and as such did not account for the critical step of particle margination to the CFL.[31] 
Particle density forces seemingly could be regarded as negligible in describing particle motion relevant to physiological blood flow, since the hydrodynamic forces due to flow (for microparticles) and Brownian forces (for nanoparticles) are often orders of magnitude higher than density-dependent body forces such as gravitational and centrifugal forces.[48,49] However, it is possible that particle density plays a role particle margination in the presence of RBCs, which will drastically affect targeting affinity from blood flow. Then, particles that have localized to the CFL will only need to travel laterally a short distance to initiate contact with the endothelium, since the CFL has a height on the order of a few micrometers. [36] Thus perhaps over the short distance of CFL thickness, density-dependent forces may be nonnegligible.

Evidence exists in the literature that density may have a role on how efficiently platelets can interact with the endothelium in blood flow. Even in the small density range reported for sub-populations of platelets (1.040-1.080 $\left.\mathrm{g} \mathrm{mL}^{-1}\right)$, it was found that the most dense platelets exhibited a roughly 4 times higher initial adhesion than the least dense platelets.[50] Materials which are proposed for diagnostics and therapeutic delivery have a much wider range of particle densities, including bubbles with densities less than blood, neutrally and non-neutrally buoyant polymers or lipid based particles, and more dense metalbased particles.[51] Therefore, it is of interest to investigate how the interaction between nanoparticles with red blood cells (RBC) affects margination when particle density is considered.

In this study, we investigate how the density-dependent forces (gravitational, centrifugal), density-independent forces (hydrodynamic, Brownian), momentum, and adhesion dynamics combined with the presence of blood components affects the targeting of $500 \mathrm{~nm}$ diameter nanospheres to inflamed endothelium from human blood flow in a parallel plate flow chamber (PPFC). This in vitro model of blood flow in a vessel is a useful tool to predict the in vivo targeting ability of a carrier system to a multitude of diseased states. In this 
work, we test the targeting ability of polystyrene, silica, and titania particles, which have densities of $1.05,2.0$, and $3.9 \mathrm{~g} \mathrm{~mL}^{-1}$, respectively. Polystyrene is used as a model drug carrier with a density comparable to typically proposed polymeric systems (such as polycaprolactone or PMMA) or neutrally buoyant liposomes. The more dense silica and titania have been proposed themselves as potential delivery vehicles or diagnostics, but can possibly also be applied to loaded liposomes or polymers which are of the same density as pure silica or titania.[31,33-35] Particles are targeted to acute inflammation for relevance to many inflammatory diseases, including atherosclerosis and arthritis. We investigate how the gravity effects change the adhesion profile of targeted spheres by orienting the chamber such that the direction of margination is either towards or away from gravity. Using a channel with a step expansion, we examine whether centrifugal force due to an induced recirculating flow region allows particles with higher densities to better target the endothelium compared to neutrally-buoyant particles.

\section{Materials and Methods}

\subsection{Preparation of vascular-targeted spheres}

Fluorescent carboxylated polystyrene spheres were purchased from Polysciences, Inc (Warrington PA). Fluorescent carboxylated silica spheres were purchased from Corpuscular, Inc (Cold Spring, NY). Amine-terminated titania spheres were purchased from EPRUI Nanoparticles \& Microparticles Co Ltd (Nanjing, China). All particles had an approximate diameter of $500 \mathrm{~nm}$. The size and zeta potential of each of the particle types used are given in

Table S1. Biotinylated sialyl Lewis A was purchased from Glycotech, Inc (Gaithersburg, MD). Biotin-phycoerythrin conjugate was purchased from Life Technologies (Grand Island, NY). Anti-cutaneous lymphocyte antigen - phycoerythrin conjugate was purchased from Miltenyi Biotec (San Diego, CA).

Carboxylated particles were conjugated first with Neutravidin Biotin Binding protein (Thermo Scientific) via a carbodiimide intermediate using EDAC (Thermo Scientific) as 
previously described.[52] Aminated particles were first conjugated with NHS-biotin, followed by conjugation with Neutravidin Biotin Binding protein overnight as with carboxylated particles. On the day of an adhesion experiment, Neutravidin conjugated particles were then conjugated with biotinylated sialyl Lewis A ( $\left.\mathrm{SLe}^{\mathrm{a}}\right)$ so that the surface density of approximately 7000 sites $\mu \mathrm{m}^{-2}$ (Table S1). Particles were then soaked for an additional 45 mins with biotin FITC to aid in post-experiment imaging. For localization experiments, particles were saturated with biotin-phycoerythrin in order to aide with imaging and prevent non-specific protein adhesion to the microchannel. Conjugation of targeting ligand to the particle surface was confirmed and quantified using an Attune $®$ Acoustic Focusing Flow Cytometer after staining with anti-cutaneous lymphocyte antigen phycoerythrin.

\subsection{HUVEC culture}

Primary HUVEC were isolated from fresh umbilical cords following a modified Jaffe protocol and cultured in tissue culture flasks as previously described by Huang et al.[53] Umbilical cords were generously donated by Mott Children's Hospital following an Internal Review Board exempt protocol. Briefly, the umbilical veins were filled with a collagenase solution for 30 mins, degrading the extracellular matrix to release the HUVEC. For adhesion assays, HUVEC were cultured onto glass coverslips, which were coated with porcine gelatin and cross-linked with glutaraldehyde before seeding.

\subsection{Blood collection and treatment}

Blood was obtained from healthy human donors via a $60 \mathrm{~mL}$ syringe containing citrate anticoagulant (acetate-citrate-dextrose, ACD) according to a protocol approved via the University of Michigan IRB and in line with standards set by the Helsinki Declaration. Human blood was collected on the day of the experiment. RBCs were isolated from other blood components via dextran sedimentation for at least 2 hours and were washed once with phosphate buffered saline (PBS) before recombining with a buffer consisting of PBS with $1 \%$ 
bovine serum albumin and $1.4 \%$ dextran. Dextran is added to this buffer so that the buffer viscosity matches human plasma viscosity.

\subsection{Parallel plate flow chamber (PPFC) adhesion assay}

Adhesion assays were performed using an in vitro model of human vasculature. The vessel construct is formed by a PPFC (Glycotech, Inc), which is an acrylic flow deck and a glass coverslip separated by a silicone gasket - the thickness of which defines the channel height (a channel height of $254 \mu \mathrm{m}$ is used for all straight channel assays). The glass coverslip is lined with a confluent HUVEC monolayer. A schematic of the PPFC setup used in this work is given in Figure 1. The HUVEC are activated for 4 hours with IL-1 $\beta$ cytokine (Fitzgerald; Acton, MA) to induce maximum expression of E-selectin. Immediately before each experiment, particles $\left(5 \times 10^{5}\right.$ particles $\left.\mathrm{mL}^{-1}\right)$ are added to a mixture of $\mathrm{RBCs}$ in buffer at $40 \%$ hematocrit, added to an inlet reservoir, and flowed through the PPFC for 5 min. Flow is driven through the PPFC to achieve a wall shear rate of $500 \mathrm{~s}^{-1}$ via a programmable syringe pump (KD Scientific; Holliston, MA) for a period of $5 \mathrm{~min}$, unless the shear rate is otherwise specified. At the end of the experiment, pure PBS with $1 \% \mathrm{BSA}$ is added to the inlet to flush the chamber and adherent particles are imaged using a Nikon Eclipse TE2000-S Brightfield Inverted Microscope equipped with a Photometric CoolSNAP EZ digital camera with a Sony CCD sensor. Adhesion assays were performed with either an upright chamber (HUVEC are on the bottom) or the chamber is inverted so that the HUVEC monolayer is at the top of the flow area. The average number of adherent particles per area of HUVEC monolayer is quantified and reported as "particle binding". The data is also reported as average number of adherent particles per endothelial cell. These numbers were calculated multiplying the particle binding by the average area occupied by an endothelial cell in our setup. The area per endothelial cell was quantified using Metamorph software, as an average over 50 cells.

A step channel was used to simulate regions of recirculation flow, typical of regions where atherosclerotic plaques are known to accumulate.[4] Figure 1B is a schematic of the 
step channel setup used for these experiments. The step channel has a pre-step channel height of $125 \mu \mathrm{m}$ and a post-step channel height of $508 \mu \mathrm{m}$. A region of recirculating flow is created immediately downstream of the step, which extends to a "reattachment" point where there is no fluid velocity at the wall parallel to the bottom of the chamber. Downstream of the reattachment point, flow redevelops into a 1-dimensional flow profile. Aside from the difference in flow pattern, the adhesion assay is carried out as described above, with the wall shear rate being calculated at the wall downstream of the step expansion. Adhesion is plotted as a function of distance from the step, and is quantified in the "disturbed flow" region (up to $1 \mathrm{~mm}$ downstream from the step) and the "far downstream" region ( $>5 \mathrm{~mm}$ downstream from the step).

\subsection{Cell-free layer localization assay}

Localization assays were performed using rectangular microchannels (sticky-Slide I 0.2 Luer, sterile) purchased from Ibidi LLC (Verona, WI) which have a channel height similar to that used in the laminar adhesion assays. Particles were mixed with $40 \%$ hematocrit RBC in buffer and flowed through the microchannel using a programmable syringe pump. The microchannel was live-imaged using an Olympus FB 1200 Confocal Microscope (compliments of Dr. Greg Thurber, University of Michigan) under 20x magnification. The shear rate was matched to that of adhesion experiments and images of particles in flow were captured from a focal plane within $2 \mu \mathrm{m}$ into the flow from the top surface of the microchannel. After thresholding the background noise, the "Analyze particles" function in ImageJ was used to automatically count the number of particles localized to the CFL.

\subsection{Statistical Analysis}

Adhesion data is obtained by manual counting of particles adherent to the endothelium after 5 minutes of blood flow. The number of adherent particles is divided by the area of the field of view (20x magnification) to give "particle binding" $\left(\# \mathrm{~mm}^{-2}\right)$. The particle binding value is multiplied by the approximate area of a single HUVEC to give "cell normalized 
binding" (particles per cell). In each experiment, at least 5 fields of view are investigated. Each experiment was performed in at least triplicate $(n \geq 3)$. Student $t$-tests were performed comparing particle adhesion to adhesion of PS or Si at the same chamber orientation. A pvalue $<0.05$ was considered statistically significant.

The "Analyze Particles" function in ImageJ is used to obtain the number of particles localized to a near-wall focal plane within each field of view. The number of adherent particles is divided by the area of the field of view to give "near-wall localization" (\# $\mathrm{mm}^{-2}$ ). Localization percent change for each particle is calculated as the ratio of the near-wall localization in blood ( $40 \%$ hematocrit in buffer) to the near-wall localization in pure buffer. In each experiment, at least 10 fields of view are investigated. Each experiment was performed in at least triplicate $(n \geq 3)$. Student t-tests were performed comparing particle localization to adhesion of PS at the same chamber orientation. A p-value $<0.05$ was considered statistically significant.

\section{Results}

An in vitro human blood flow assay is used to study the targeting ability of polystyrene (PS), silica (Si), and titania (Ti) spheres to the vascular wall in various blood flow conditions. The PS, Si, and Ti spheres have approximately $500 \mathrm{~nm}$ diameter and densities of $1.05,2.0$, and $3.9 \mathrm{~g} \mathrm{~cm}^{-3}$, respectively. Particles are surface-coated with $\mathrm{sLe}^{\mathrm{A}}$ targeting ligand and flowed either in simple buffer or a RBC-in-buffer mixture through a PPFC lined with an activated (inflamed) monolayer of HUVECs. Parameters including shear rate, blood flow profile, chamber orientation and microchannel height are altered to determine how particle density affects targeting affinity.

\subsection{Effect of RBCs on Particle Adhesion in Laminar Flow}

Figure 2 compares the adhesion density of targeted PS, Si, and Ti nanospheres following 5 min of either buffer flow or RBC-in-buffer (see section 2.3) flow over an activated HUVEC monolayer at a physiological shear rate of $500 \mathrm{~s}^{-1}$. Representative images 
of particles bound on HUVEC are shown in Figure S1. In buffer flow (Figure 2A), particle adhesion to HUVEC at the bottom of the chamber increases with particle density, as $\mathrm{Si}$ and $\mathrm{Ti}$ spheres adhered 360\% and 640\% higher, respectively, than PS spheres (though the difference for Si and PS was not statistically significant).

Multiple interesting observations can be made when a physiologically relevant number of RBCs are added to the experimental setup (Figure 2B). First, we see that the addition of $\mathrm{RBCs}$ results in an increase in the number of adherent particles for each particle type compared to buffer flow. When the HUVEC monolayer is at the bottom of the chamber, the adhesion of $\mathrm{Si}$ and $\mathrm{Ti}$ spheres is $257 \%$ and $91 \%$ higher, respectively, than that of PS spheres. When the chamber is inverted and binding is occurring against the direction of gravity, the adhesion of Si spheres is still $236 \%$ higher than that of PS spheres, while the adhesion densities of PS and Ti spheres are not significantly different. The fact that Si particles still bind more than neutrally-buoyant PS in the inverted chamber suggests that more Si spheres are available at the endothelium for binding in the presence of $\mathrm{RBC}$, particularly given that the average adhesion in upright buffer flow assays (Figure 2A) increased with increasing particle density.

\subsection{Localization of Particles to the Cell-Free Layer}

In light of the observations seen in Figure 2, it is important to consider what mechanisms may cause the density-dependent differences in adhesion in the presence of RBCs. One possible behavior that could result in improved adhesion to the endothelium would be the improved localization of particles to the CFL, resulting in a higher number of particles with an opportunity to bind. In order to investigate this, we utilize a previously described microchannel setup in which fluorescent particles localized to a near-wall focal plane are imaged using a confocal microscope.[38,39] This setup decouples particle localization from the ligand/receptor adhesion to determine how the presence of RBCs affects only localization to the near-wall region. As an upright confocal microscope was used, 
particles localized near the upper wall of the flow chamber are imaged. Figure 3A shows the near-wall (within $2 \mu \mathrm{m}$ distance from top wall) localization of PS, Si, and Ti particles from RBC-in-buffer flow in a microchannel under the same conditions as the adhesion experiment represented by Figure 1B (inverted). On average, the Si particles localized to the CFL in the presence of RBCs the most, followed by PS and then Ti particles (differences were not significant). We then compared the near-wall localization in RBCs-in buffer flow to the nearwall localization in the absence of RBCs (Figure 3B). Interestingly, the addition of RBCs results in a $13 \%$ increase in the amount of Si spheres localized to the near-wall region compared to particles in buffer flow, while adding RBCs results in a $12 \%$ and $4 \%$ decrease in the amount of near-wall localized PS and Ti spheres relative to buffer flow.

\subsection{Effect of Shear Rate on Adhesion of Dense Particles}

Evidence based on the results shown in Figures 2 and 3 suggests that Si spheres have higher adhesion from RBC-in-buffer flow than Ti spheres because Si are more effective at localizing to the CFL in the presence of RBCs. Another factor which must be considered in the adhesion experiment once particles are in the CFL is the binding kinetics between the targeting ligands on the particle and the targeted receptors, countered by the fact that these particles carry translational momentum that the ligand/receptor interaction must overcome to result in firm adhesion. Figure 4 shows the adhesion level of Si and Ti spheres to inflamed endothelium at a range of laminar shear rates (from 50-1000 s $\mathrm{s}^{-1}$ ) for a fixed experiment time of $5 \mathrm{~min}$. At the lowest shear rates investigated $\left(50,100 \mathrm{~s}^{-1}\right)$, Si and Ti display the same adhesion. As the shear rates increased from 100 to $1000 \mathrm{~s}^{-1}$, the adhesion of Si spheres increases to a maximum at $500 \mathrm{~s}^{-1}$; followed by a significant decrease in adhesion at $1000 \mathrm{~s}^{-1}$. However, at shear rates above $100 \mathrm{~s}^{-1}$, the Ti particles do not show a similar increase in adhesion as was seen for Si particles, which resulted in Si particles displaying significantly higher adhesion than the Ti particles at the intermediate shear rates. When the adhesion data is normalized to the number of particles used per experiment (Figure 4B), so that the 
"adhesion efficiency" of particles at different shear rates can be properly compared, both Si and Ti particles are found to exhibit reduced adhesion efficiency as the shear rate increases from $100-1000 \mathrm{~s}^{-1}$. However, the adhesion efficiency for Ti particles decreases more than $\mathrm{Si}$ particles particularly at 200 and $500 \mathrm{~s}^{-1}$. At $1000 \mathrm{~s}^{-1}$, the adhesion efficiency of Si spheres decreases to a level not different from that of Ti spheres.

\subsection{Effect of Recirculation Flow on Adhesion}

Some chronic inflammatory diseases including atherosclerosis are known to form in areas of the vasculature characterized by disturbed flow profiles (recirculating flow or oscillating pulsatility) caused by curvature or bifurcations in the vessel.[54,55] The presence of a permanent recirculating flow region introduces density-dependent centrifugal force, which may alter the adhesion profile in these areas. We investigate this type of disturbed blood flow pattern using a modified PPFC setup, in which a vertical step in the channel causes a recirculation zone to form immediately downstream of the step.[56] Further down from the step is the a reattachment point (a point of flow separation) where a portion of the flow streamline moves downstream toward steady laminar flow, and a far downstream region is defined where the flow is redeveloped into a steady laminar profile. We then can observe the particle adhesion profile to a layer of inflamed endothelium adjacent to this recirculation eddy, as well as in the far downstream region.

Figure 5 shows the adhesion of targeted PS, Si, and Ti spheres to activated HUVEC from recirculating blood flow. At this shear rate $\left(200 \mathrm{~s}^{-1}\right.$, as calculated from the expanded section of the chamber), the recirculation zone is roughly $225 \mu \mathrm{m}$ from the step to the reattachment point (the point at which flow separates). It is apparent, particularly for Si and Ti particles, that adhesion then is a function of distance downstream of the step. Adhesion within the recirculation region is increased for $\mathrm{Si}$ and Ti particles compared to PS, whether the chamber is in the upright or inverted orientation. The adhesion for Si and Ti spheres is also higher than for PS spheres in the far downstream region when the chamber is upright, but 
there is no significant difference between particle types far downstream when the chamber is inverted. Also, the adhesion of $\mathrm{Si}$ and $\mathrm{Ti}$ in the recirculating flow pattern and further downstream is not significantly different, suggesting that the two particles are distributed to the wall area about the same.

It should be noted that the adhesion in the recirculation channel is not directly comparable to the laminar flow experiments in Figure 2, which were done at a higher shear rate and in a channel with a smaller height. A separate set of laminar flow assays were done at the same shear rate and channel height $\left(200 \mathrm{~s}^{-1}, 508 \mu \mathrm{m}\right)$ as the recirculation flow assay, in order to elucidate the effect of recirculation flow on adhesion in the far downstream region of the channel (Figure S2). In the recirculation region and in the far downstream region in an upright channel, adhesion for $\mathrm{Si}$ and $\mathrm{Ti}$ spheres is roughly twice that of the adhesion in steady laminar flow in a straight channel, while PS spheres is about the same regardless of flow type. When the chamber is inverted, there is no difference in particle adhesion from laminar flow with upstream recirculation and laminar flow in a straight channel.

Since the improved adhesion of dense nanoparticles in recirculation flow diminishes in an inverted channel, it may be that the negative gravity component overrides any benefit of the recirculation flow. Then, it is of interest to examine whether the adhesion in the inverted channel will improve if the magnitude of the centrifugal force is increased. To test this we examined the adhesion of PS, Si, and Ti particles from recirculation blood flow at a higher shear rate of $625 \mathrm{~s}^{-1}$, which increases the magnitude of centrifugal force present. Indeed, we find that at this higher shear rate, the Si spheres appear to be less sensitive to gravity force due to the chamber being inverted compared to at $200 \mathrm{~s}^{-1}$ (Figure 6). Inverting the chamber at $200 \mathrm{~s}^{-1}$ results in a 55\% and $68 \%$ decrease in binding of Si spheres in the disturbed region and far downstream, respectively, compared to the upright chamber. At $625 \mathrm{~s}^{-1}$, these decreases in Si adhesion are only $25 \%$ and $28 \%$, suggesting that the increased shear makes adhesion less dependent on chamber orientation. The PS particles which are essentially neutrally-buoyant 
show no dependence on chamber orientation, while the Ti particles only show decreased binding in the far downstream region of the inverted chamber.

\section{Discussion}

The design of vascular targeted carriers (VTCs) has garnered significant attention recent years because of the potential to achieve highly localized delivery to diseased tissues. It has become clear that the physical parameters, such as particle size and shape, are important in determining the ability of VTCs to marginate and adhere to the vascular wall from blood flow.[1-5,38,39,57] In light of the wide variety of carrier types proposed, particle density is a relevant parameter to explore for its potential effects on margination and adhesion. Particle density has been shown in simple buffer flow to affect margination[31], however it remains unclear how particle density affects margination in blood flow. Nanoparticles which are neutrally-buoyant have been shown to marginate inefficiently in the presence of RBCs, making them sub-optimal as VTCs.[1-5,38,39,57] In this work, we attempt to directly compare the margination in blood and adhesion to HUVEC of neutrally-buoyant particles with particles that are twice and four times as dense to determine what effect, if any, density has on targeting.

The results of this study show that particles with different density display differences in targeting ability in buffer flow and particularly in the presence of RBCs. The fact that adhesion increases with particle density in pure buffer flow is an initial indication that gravity may play a role in particle margination at this particle size. This is in contrast to a previous study which showed that in buffer flow, increased density had a negative effect on margination of nanospheres with diameters ranging from 60-65 nanometers.[31] However, these contrasting results may potentially be due to the different particle sizes used for experiments. The gravity component for the $65 \mathrm{~nm}$ spheres is neglected and the decreased margination is attributed to the increased particle momentum competing with Brownian motion toward the chamber wall. The $500 \mathrm{~nm}$ nanospheres used here will have a larger 
gravity force, which could contribute to their margination to the bottom of the chamber compared to the smaller $(60-65 \mathrm{~nm})$ nanospheres rather than being negligible.

The addition of RBCs results in the increased adhesion of each particle type compared to in pure buffer flow. This is not surprising, as RBCs are known to improve adhesion kinetics for cells/particles by providing an additional force normal to the endothelium which helps counteract the shear force imparted by blood flow that would act to remove adherent cells from the surface.[58] What we find here, however, is that this RBC effect on adhesion is not the same for particles with different densities. Si spheres displayed significantly greater adhesion than either PS or Ti spheres, despite Ti spheres displaying the greatest adhesion in buffer flow. One possibility for this increased adhesion is a difference in the number of particles localized to channel wall, providing more particles an opportunity to bind. Indeed, Si spheres on average localized close to the wall in higher amounts than either PS or Ti spheres (though differences were not significant). Also, the addition of RBCs elicited increased near-wall localization of Si spheres compared to in pure buffer flow, while RBCs actually decreased the localization of both PS and Ti spheres relative to buffer. Overall, the combination of improved localization along with the RBC-aided adhesion potentially explains why the Si spheres in Figure 2 exhibited disproportionately higher adhesion than both PS and Ti spheres. While different material chemistries can have effect on particle behavior in suspending medium and interactions with the endothelial cell membrane[59], we expect that the primary force governing the particle adhesion is the specific ligand-receptor interaction rather than other non-specific forces, because there is zero particle adhesion to the endothelium when there are no targeting ligands present on the particle surfaces (data not shown). Also, the particles coated with targeting ligand displayed similar zeta potentials in the adhesion assay buffer (Table S1). In our system we do not expect that differences in material type are affecting adhesion results due to both the high coverage of targeting ligand used, and the similar levels of adhesion shown in the buffer flow experiment (Figure 2A). 
Still, in a more physiological environment (e.g. the inclusion of blood plasma), the effects of using different particle materials may be more pronounced.

It is known that parameters such as shape, size, and membrane rigidity can affect induced migration of particles/cells in blood; and we see here evidence that density may play a role as well. The question that remains is why do Ti particles exhibit lower localization (and hence lower adhesion) than Si particles despite their significantly higher density? Recent reports by Kumar and Graham show that heterogeneous collisions between deformable RBCs and more rigid cells/particles contribute to the margination of the more rigid bodies to the CFL. This occurs because the rigid particles experience a substantial displacement upon collision with the larger deformable RBCs.[60-62] It is possible that density neutral and slightly dense particles such as PS and Si are easily displaced in this manner with the latter of the two experiencing a greater displacement due to larger momentum. However, the Ti particles (which are 4 times as dense) may require larger force from RBC impact to displace it from its original trajectory.

Another possibility is the presence of inertial lift forces acting on particles within microchannel flow. It is well described that in microchannel flow, inertial lift forces arising from the parabolic flow profile (as is present in our setup) can result in the lateral migration of particles across streamlines, even at relatively low Reynolds numbers.[63] We suspect that these forces may be at play in our setup, though the situation is made more complex by the presence of RBCs. Estimated from buffer flow, lateral migration velocity should be on the order of nanometers/sec, which seems negligible, however if occurring within the CFL could potentially result in migrations toward the endothelium. Also acting on the particles is a wall induced lift force that acts to push particles away from the wall when the particle is sufficiently close. In all, particle motion in the CFL may be governed by a combination on inertial and wall lift force, gravity forces, momentum, and collisions with RBCs acting to keep particles in the CFL. Particles with different density will have different Stokes numbers, 
and thus different propensities to move across streamlines. It is possible that particles will have different tendencies to collide with the endothelium once in the CFL, though the Stokes numbers calculated within our channel are still $<1$ which indicates a propensity to follow streamlines. Overall, it seems the major difference causing differences in adhesion lies with the interactions with RBCs causing different localization levels to the CFL.

Another factor to consider is the fact that particles with different density will carry different translational momentum which could affect the ligand/receptor adhesion. We know from previous works that there exists a "critical shear rate" (CSR), above which the resulting particle adhesion decreases with increasing shear rate - due to transition of particle binding into a reaction-limited regime.[3] This CSR is highly dependent on particle diameter, as increasing particle size increases the shear removal force felt by a particle in blood flow. For a given particle size, there is a shear rate above which adhesion is limited by the adhesion kinetics being overwhelmed by the shear removal forces. We see evidence in this work that this CSR may be density-dependent as well. This makes sense, as at a given shear rate and ligand surface coverage, a Ti particle would need to overcome a higher force than a Si particle to firmly adhere. We do see that the adhesion efficiency for both particle types was found to consistently decrease with increasing shear rate, which is likely because of the corresponding increase in translational momentum competing with adhesion forces. This difference in the adhesion profile for particles with different density is an interesting observation, particularly when considering that the vasculature contains a wide range of local shear rates and blood flow profiles. Thus, it may well be that the optimal targeting of specific areas of the vasculature requires a particle type with a specific density, depending on the local hemodynamics and blood flow profile. It is also important to note that the apparent CSRs observed in this study are specific to the experimental protocol used, and that CSR could change based on experimental variables that could affect the hemodynamics or reaction kinetics such as targeting ligand density, channel height, hematocrit, flow profile, etc. 
The addition of centrifugal force via induced recirculation flow had noticeable effects on non-neutrally buoyant particles, particularly within the disturbed flow region in the immediate vicinity of the recirculation zone. In a previous work, we use this setup to examine the adhesion pattern of neutrally-buoyant spheres and rods and found no improvement due to shape within the recirculation region.[39] Interestingly, we do see increased adhesion of more dense particles in this region. This increase in adhesion is most pronounced when gravity is also acting on the particles in the direction of the HUVEC monolayer. The higher adhesion of Si and Ti particles compared to PS in the disturbed region with gravity opposite the direction of the HUVEC suggests that the presence of the recirculation flow is causing increased redistribution of $\mathrm{Si}$ and $\mathrm{Ti}$ particles to the CFL and thus the HUVEC monolayer. This observation is further supported by the observation that non-neutrally buoyant particles exhibit increased adhesion in the recirculation flow compared to laminar flow (comparing Figure 5 to Figure S2). In an inverted channel, this effect is diminished in the far downstream region - showing that gravity effects still persist. By increasing the shear rate (Figure 6), thus increasing the contribution due to centrifugal force, we find the adhesion of Si spheres is less dependent on chamber orientation. These results suggest that the combination of particle density-dependent gravity and centrifugal forces can both affect localization and subsequent adhesion to the endothelium depending on local hemodynamics, which can vary depending on which area of the vasculature is targeted.

With any drug delivery system formulation, the risk of causing adverse effects is a serious consideration. Accumulation of the particulate delivery systems in unwanted areas of the vasculature due to mechanical entrapment in small capillaries, filtration by organs of the RES, or adhesion to non-targeted endothelium are issues which could reduce the efficacy of the carrier system. The $500 \mathrm{~nm}$ particle size used in this study is relevant for drug delivery in that spheres with diameters on the order of $200 \mathrm{~nm}$ have typically been proposed for use as VTCs (e.g. $250 \mathrm{~nm}$ in [64]; $190 \mathrm{~nm}$ in [65]; $330 \mathrm{~nm}$ in [66]), and we have shown in several 
publications that $200 \mathrm{~nm}$ and $500 \mathrm{~nm}$ spheres exhibit similar margination and binding dynamics under human blood flow conditions.[4,38] Moreover, VTCs in the $200-500 \mathrm{~nm}$ have previously been evaluated in vivo in mice with no significant adverse effects reported.[4,57,64] However, an important consideration associated with this study is whether high-velocity collisions of dense particles with the vascular wall could potentially cause adverse effects on the endothelium itself. This may be particularly important for targeting to late-stage atherosclerosis, as significant disruptions in the endothelium could potentially lead to clot formation or trigger an event. The short-term in vitro experiments performed in this study did not elucidate any obvious endothelial damage, and to our knowledge there is no indication of this type of endothelial damage reported in the literature. Still, this is an important question to keep in mind, which could limit the feasibility of these types of drug delivery systems; particularly for particles with ultra high density (e.g. gold nanoparticles).

\section{Conclusion}

Particle density, along with local hemodynamic conditions including shear rate, flow type, channel height, and the presence of RBCs, all affect how well targeted nanosized carrier systems adhere to the target site. We have shown previously that neutrally-buoyant PS spheres on the sub-micron and nanoscale do not adhere to endothelium in the presence of RBCs as efficiently as microspheres. Spheres of this size range are not preferentially distributed to the CFL in the presence of RBCs, as is known to occur for microparticles and other micron scale entities such as leukocytes and platelets. In this study, Si particles with a density roughly twice that of blood displayed increased adhesion to endothelium relative to the neutrally-buoyant particles of same size (500 nm diameter) when in the presence of RBCs. The improved adhesion of Si particles is likely linked to increased localization to the CFL in blood flow, despite having a diameter smaller than that which provides improved CFL localization for neutrally-buoyant spheres. This work suggests that density is a parameter which can be used to improve targeting ability of nanoparticles to the vascular wall, and the 
wide variety of carrier types that have been proposed as drug delivery systems makes this a possibility.

\section{Acknowledgements}

The authors would like to thank the NSF (CBET1054352) for funding provided to complete this work.

\section{Disclosures}

The authors have no conflicts of interest.

[1] Tilles AW, Eckstein EC. The near-wall excess of platelet-sized particles in blood flow: its dependence on hematocrit and wall shear rate. Microvasc Res 1987;33:211-23. doi:10.1016/0026-2862(87)90018-5.

[2] Eckstein EC, Tilles AW, Millero 3rd FJ. Conditions for the occurrence of large nearwall excesses of small particles during blood flow. Microvasc Res 1988;36:31-9.

[3] Charoenphol P, Huang RB, Eniola-Adefeso O. Potential role of size and hemodynamics in the efficacy of vascular-targeted spherical drug carriers. Biomaterials 2010;31:1392-402. doi:10.1016/j.biomaterials.2009.11.007.

[4] Charoenphol P, Mocherla S, Bouis D, Namdee K, Pinsky DJ, Eniola-Adefeso O. Targeting therapeutics to the vascular wall in atherosclerosis--carrier size matters. Atherosclerosis 2011;217:364-70. doi:10.1016/j.atherosclerosis.2011.04.016.

[5] Lee TR, Choi M, Kopacz AM, Yun SH, Liu WK, Decuzzi P. On the near-wall accumulation of injectable particles in the microcirculation: smaller is not better. Sci Rep 2013;3:2079. doi:10.1038/srep02079.

[6] Howard M, Zern BJ, Anselmo AC, Shuvaev V V., Mitragotri S, Muzykantov V. Vascular targeting of nanocarriers: Perplexing aspects of the seemingly straightforward paradigm. ACS Nano 2014;8:4100-32. doi:10.1021/nn500136z.

[7] Eniola-Adefeso O, Heslinga MJ, Porter TM. Design of nanovectors for therapy and imaging of cardiovascular diseases. Methodist Debakey Cardiovasc J 2012;8:13-7.

[8] Unger E, Porter T, Lindner J, Grayburn P. Cardiovascular drug delivery with ultrasound and microbubbles. Adv Drug Deliv Rev 2014;72:110-26. doi:10.1016/j.addr.2014.01.012.

[9] Ting CY, Fan CH, Liu HL, Huang CY, Hsieh HY, Yen TC, et al. Concurrent bloodbrain barrier opening and local drug delivery using drug-carrying microbubbles and focused ultrasound for brain glioma treatment. Biomaterials 2012;33:704-12. doi:10.1016/j.biomaterials.2011.09.096. 
[10] Nance E, Zhang C, Shih T-Y, Xu Q, Schuster BS, Hanes J. Brain-Penetrating Nanoparticles Improve Paclitaxel Efficacy in Malignant Glioma Following Local Administration. ACS Nano 2014. doi:10.1021/nn504210g.

[11] Shuvaev V V., Ilies MA, Simone E, Zaitsev S, Kim Y, Cai S, et al. Endothelial targeting of antibody-decorated polymeric filomicelles. ACS Nano 2011;5:6991-9. doi:10.1021/nn2015453.

[12] Kamaly N, Xiao Z, Valencia PM, Radovic-Moreno AF, Farokhzad OC. Targeted polymeric therapeutic nanoparticles: design, development and clinical translation. Chem Soc Rev 2012;41:2971. doi:10.1039/c2cs15344k.

[13] Muro S, Dziubla T, Qiu W, Leferovich J, Cui X, Berk E, et al. Endothelial targeting of high-affinity multivalent polymer nanocarriers directed to intercellular adhesion molecule 1. J Pharmacol Exp Ther 2006;317:1161-9. doi:10.1124/jpet.105.098970.bosis.

[14] Allen TM. Long-circulating (sterically stabilized) liposomes for targeted drug delivery. Trends Pharmacol Sci 1994;15:215-20. doi:10.1016/0165-6147(94)90314-X.

[15] Allen TM, Cullis PR. Liposomal drug delivery systems: From concept to clinical applications. Adv Drug Deliv Rev 2013;65:36-48. doi:10.1016/j.addr.2012.09.037.

[16] Liong M, Lu J, Kovochich M, Xia T, Ruehm SG, Nel AE, et al. Multifunctional inorganic nanoparticles for imaging, targeting, and drug delivery. ACS Nano 2008;2:889-96. doi:10.1021/nn800072t.

[17] Huang H-C, Barua S, Sharma G, Dey SK, Rege K. Inorganic nanoparticles for cancer imaging and therapy. J Control Release 2011;155:344-57. doi:10.1016/j.jconrel.2011.06.004.

[18] Tom RT, Suryanarayanan V, Reddy PG, Baskaran S, Pradeep T. Ciprofloxacinprotected gold nanoparticles. Langmuir 2004;20:1909-14. doi:10.1021/la0358567.

[19] Samokhin G, Smirnov M, Muzykantov V, Domogatsky S, Smirnov V. Effect of flow rate and blood cellular elements on the efficiency of red blood cell targeting to collagen-coated surfaces. J Appl Biochem 1984;6:70-5.

[20] Muzykantov VR. Drug delivery by red blood cells: vascular carriers designed by Mother Nature. Expert Opin Drug Deliv 2011;7:403-27. doi:10.1517/17425241003610633.Drug.

[21] Park J Il, Jagadeesan D, Williams R, Oakden W, Chung S, Stanisz GJ, et al. Microbubbles loaded with nanoparticles: A route to multiple imaging modalities. ACS Nano 2010;4:6579-86. doi:10.1021/nn102248g.

[22] Anselmo AC, Mitragotri S. Cell-mediated delivery of nanoparticles: Taking advantage of circulatory cells to target nanoparticles. J Control Release 2014. doi:10.1016/j.jconrel.2014.03.050. 
[23] Geers B, Lentacker I, Sanders NN, Demeester J, Meairs S, De Smedt SC. Selfassembled liposome-loaded microbubbles: The missing link for safe and efficient ultrasound triggered drug-delivery. J Control Release 2011;152:249-56. doi:10.1016/j.jconrel.2011.02.024.

[24] Bertini I, Bianchini F, Calorini L, Colagrande S, Fragai M, Franchi A, et al. Persistent contrast enhancement by sterically stabilized paramagnetic liposomes in murine melanoma. Magn Reson Med 2004;52:669-72. doi:10.1002/mrm.20189.

[25] Park SH, Oh SG, Mun JY, Han SS. Loading of gold nanoparticles inside the DPPC bilayers of liposome and their effects on membrane fluidities. Colloids Surfaces B Biointerfaces 2006;48:112-8. doi:10.1016/j.colsurfb.2006.01.006.

[26] Ito A, Kuga Y, Honda H, Kikkawa H, Horiuchi A, Watanabe Y, et al. Magnetite nanoparticle-loaded anti-HER2 immunoliposomes for combination of antibody therapy with hyperthermia. Cancer Lett 2004;212:167-75. doi:10.1016/j.canlet.2004.03.038.

[27] Kikumori T, Kobayashi T, Sawaki M, Imai T. Anti-cancer effect of hyperthermia on breast cancer by magnetite nanoparticle-loaded anti-HER 2 immunoliposomes. Breast Cancer Res Treat 2009;113:435-41. doi:10.1007/s10549-008-9948-x.

[28] Cherukuri P, Glazer ES, Curley SA. Targeted hyperthermia using metal nanoparticles. Adv Drug Deliv Rev 2010;62:339-45. doi:10.1016/j.addr.2009.11.006.

[29] Liu S, Zhang Z, Han MY. Nanometer-sized gold-loaded gelatin/silica nanocapsules. Adv Mater 2005;17:1862-6. doi:10.1002/adma.200500124.

[30] Takalkar AM, Klibanov AL, Rychak JJ, Lindner JR, Ley K. Binding and detachment dynamics of microbubbles targeted to P-selectin under controlled shear flow. J Control Release 2004;96:473-82. doi:10.1016/j.jconrel.2004.03.002.

[31] Toy R, Hayden E, Shoup C, Baskaran H, Karathanasis E. The effects of particle size, density and shape on margination of nanoparticles in microcirculation. Nanotechnology 2011;22:115101. doi:10.1088/0957-4484/22/11/115101.

[32] Singh AK, Cummings EB, Throckmorton DJ. Fluorescent liposome flow markers for microscale particle-image velocimetry. Anal Chem 2001;73:1057-61. doi:10.1021/ac001159x.

[33] Koo OM, Rubinstein I, Onyuksel H. Role of nanotechnology in targeted drug delivery and imaging: a concise review. Nanomedicine Nanotechnology, Biol Med 2005;1:193212. doi:10.1016/j.nano.2005.06.004.

[34] Barbé C, Bartlett J, Kong L, Finnie K, Lin HQ, Larkin M, et al. Silica particles: A novel drug-delivery system. Adv Mater 2004;16:1959-66. doi:10.1002/adma.200400771.

[35] Sahoo SK, Labhasetwar V. Nanotech approaches to drug delivery and imaging. Drug Discov Today 2003;8:1112-20. doi:10.1016/S1359-6446(03)02903-9. 
[36] Migliorini C, Qian YH, Chen HD, Brown EB, Jain RK, Munn LL. Red blood cells augment leukocyte rolling in a virtual blood vessel. Biophys J 2002;83:1834-41.

[37] Nobis U, Pries AR, Cokelet GR, Gaehtgens P. Radial distribution of white cells during blood flow in small tubes. Microvasc Res 1985;29:295-304.

[38] Namdee K, Thompson AJ, Charoenphol P, Eniola-Adefeso O. Margination propensity of vascular-targeted spheres from blood flow in a microfluidic model of human microvessels. Langmuir 2013;29:2530-5. doi:10.1021/la304746p.

[39] Thompson AJ, Mastria EM, Eniola-Adefeso O. The margination propensity of ellipsoidal micro/nanoparticles to the endothelium in human blood flow. Biomaterials 2013;34:5863-71. doi:10.1016/j.biomaterials.2013.04.011.

[40] Nicolas J, Mura S, Brambilla D, Mackiewicz N, Couvreur P. Design, functionalization strategies and biomedical applications of targeted biodegradable/biocompatible polymer-based nanocarriers for drug delivery. Chem Soc Rev 2013;42:1147-235. doi:10.1039/c2cs35265f.

[41] Petros RA, DeSimone JM. Strategies in the design of nanoparticles for therapeutic applications. Nat Rev Drug Discov 2010;9:615-27. doi:10.1038/nrd2591.

[42] Parveen S, Misra R, Sahoo SK. Nanoparticles: A boon to drug delivery, therapeutics, diagnostics and imaging. Nanomedicine Nanotechnology, Biol Med 2012;8:147-66. doi:10.1016/j.nano.2011.05.016.

[43] Muro S. Challenges in design and characterization of ligand-targeted drug delivery systems. J Control Release 2012;164:125-37. doi:10.1016/j.jconrel.2012.05.052.

[44] Adriani G, de Tullio MD, Ferrari M, Hussain F, Pascazio G, Liu X, et al. The preferential targeting of the diseased microvasculature by disk-like particles. Biomaterials 2012;33:5504-13. doi:10.1016/j.biomaterials.2012.04.027.

[45] Chacko AM, Hood ED, Zern BJ, Muzykantov VR. Targeted nanocarriers for imaging and therapy of vascular inflammation. Curr Opin Colloid Interface Sci 2011;16:21527. doi:10.1016/j.cocis.2011.01.008.

[46] Muro S, Garnacho C, Champion JA, Leferovich J, Gajewski C, Schuchman EH, et al. Control of endothelial targeting and intracellular delivery of therapeutic enzymes by modulating the size and shape of ICAM-1-targeted carriers. Mol Ther 2008;16:1450-8. doi:10.1038/mt.2008.127.

[47] Gentile F, Curcio A, Indolfi C, Ferrari M, Decuzzi P. The margination propensity of spherical particles for vascular targeting in the microcirculation. J Nanobiotechnology 2008;6:9. doi:10.1186/1477-3155-6-9.

[48] Lee SY, Ferrari M, Decuzzi P. Shaping nano-/micro-particles for enhanced vascular interaction in laminar flows. Nanotechnology 2009;20:495101. doi:10.1088/09574484/20/49/495101. 
[49] Jurney P, Agarwal R, Singh V, Roy K, Sreenivasan S V, Shi L. The Effect of Nanoparticle Size on Margination and Adhesion Propensity in Artificial MicroCapillaries. Proc Asme Micro/Nanoscale Heat Mass Transf Int Conf 2012 2012:109_ 15.

[50] Polanowskagrabowska R, Raha S, Gear ARL. Adhesion Efficiency, Platelet Density and Size. Br J Haematol 1992;82:715-20. doi:DOI 10.1111/j.13652141.1992.tb06949.x.

[51] Doshi N, Mitragotri S. Designer biomaterials for nanomedicine. Adv Funct Mater 2009;19:3843-54. doi:10.1002/adfm.200901538.

[52] Eniola AO, Hammer DA. Characterization of biodegradable drug delivery vehicles with the adhesive properties of leukocytes II: Effect of degradation on targeting activity. Biomaterials 2005;26:661-70. doi:10.1016/j.biomaterials.2004.03.003.

[53] Huang RB, Eniola-Adefeso O. Shear stress modulation of IL-1beta-induced E-selectin expression in human endothelial cells. PLoS One 2012;7:e31874. doi:10.1371/journal.pone.0031874.

[54] Malek AM, Alper SL, Izumo S. Hemodynamic shear stress and its role in atherosclerosis. JAMA 1999;282:2035-42. doi:10.1001/jama.282.21.2035.

[55] Nguyen ND, Haque AK. Effect of hemodynamic factors on atherosclerosis in the abdominal aorta. Atherosclerosis 1990;84:33-9. doi:10.1016/0021-9150(90)90005-4.

[56] Charoenphol P, Onyskiw PJ, Carrasco-Teja M, Eniola-Adefeso O. Particle-cell dynamics in human blood flow: implications for vascular-targeted drug delivery. $\mathbf{J}$ Biomech 2012;45:2822-8. doi:10.1016/j.jbiomech.2012.08.035.

[57] Namdee K, Thompson AJ, Golinski A, Mocherla S, Bouis D, Eniola-Adefeso O. In vivo evaluation of vascular-targeted spheroidal microparticles for imaging and drug delivery application in atherosclerosis. Atherosclerosis 2014;237:279-86. doi:10.1016/j.atherosclerosis.2014.09.025.

[58] Migliorini C, Qian Y, Chen H, Brown EB, Jain RK, Munn LL. Red blood cells augment leukocyte rolling in a virtual blood vessel. Biophys J 2002;83:1834-41. doi:10.1016/S0006-3495(02)73948-9.

[59] Nel AE, Mädler L, Velegol D, Xia T, Hoek EM V, Somasundaran P, et al. Understanding biophysicochemical interactions at the nano-bio interface. Nat Mater 2009;8:543-57. doi:10.1038/nmat2442.

[60] Kumar A, Graham MD. Mechanism of margination in confined flows of blood and other multicomponent suspensions. Phys Rev Lett 2012;109.

doi:10.1103/PhysRevLett.109.108102.

[61] Kumar A, Graham MD. Margination and segregation in confined flows of blood and other multicomponent suspensions. Soft Matter 2012;8:10536.

doi:10.1039/c2sm25943e. 
[62] Kumar A, Graham MD. Segregation by membrane rigidity in flowing binary suspensions of elastic capsules. Phys Rev E - Stat Nonlinear, Soft Matter Phys 2011;84. doi:10.1103/PhysRevE.84.066316.

[63] Di Carlo D. Inertial microfluidics. Lab Chip 2009;9:3038-46. doi:10.1039/b912547g.

[64] Li JM, Wang YY, Zhang W, Su H, Ji LN, Mao ZW. Low-weight polyethylenimine cross-linked 2-hydroxypopyl- $\beta$-cyclodextrin and folic acid as an efficient and nontoxic siRNA carrier for gene silencing and tumor inhibition by VEGF siRNA. Int J Nanomedicine 2013;8:2101-17. doi:10.2147/IJN.S42440.

[65] Marrache S, Dhar S. Biodegradable synthetic high-density lipoprotein nanoparticles for atherosclerosis. Proc Natl Acad Sci U S A 2013;110:9445-50.

doi:10.1073/pnas.1301929110.

[66] Yang H, Zhao F, Li Y, Xu M, Li L, Wu C, et al. VCAM-1-targeted core/shell nanoparticles for selective adhesion and delivery to endothelial cells with lipopolysaccharide-induced inflammation under shear flow and cellular magnetic resonance imaging in vitro. Int J Nanomedicine 2013;8:1897-906. doi:10.2147/IJN.S44997. 


\section{Captions}

Fig. 1 Schematic of the parallel plate flow chamber (PPFC) setup used for laminar flow (A) and recirculation flow (B) assays. The top of the chamber is formed by an acrylic flow deck and the bottom of the chamber is formed by a glass coverslip on which a HUVEC monolayer has been cultured. The gasket which is sandwiched between the flow deck and coverslip defines the flow area. Recirculation flow is achieved by using two gaskets to create a step expansion downstream of the inlet.

Fig. 2 Adhesion of sLe ${ }^{\mathrm{a}}$-coated PS, Si, and Ti spheres (500 nm diameter) to inflamed endothelium after 5 minutes of particle-loaded $\left(5 \times 10^{5}\right.$ particles $/ \mathrm{mL}$ total volume) buffer flow (A) or RBC-in-buffer flow (B) at a shear rate of $500 \mathrm{~s}^{-1}$. Experiments were done with the endothelial layer at the bottom (upright) of the chamber in the buffer flow experiments, and chamber orientation was varied in the RBC-in-buffer flow experiments. Particle binding is reported as the number of adherent particles per area. Cell normalized binding is reported as the number of adherent particles per endothelial cell (EC). Student t-tests were performed comparing particle adhesion to adhesion of $* \mathrm{PS}$ or ${ }^{\#} \mathrm{Si}$ at the same chamber orientation. A pvalue $<0.05$ was considered statistically significant.

Fig. 3 (A) Localization of PS, Si, and Ti spheres (500 nm diameter) to within $2 \mu \mathrm{m}$ of the upper wall of a microchannel from particle-loaded RBC-in-buffer flow $\left(5 \times 10^{5}\right.$ particles $/ \mathrm{mL}$ total volume) at $500 \mathrm{~s}^{-1}$, and (B) percent change in particle localization comparing localization in particle-loaded buffer flow and particle loaded RBC-in-buffer flow under the same conditions. Student t-tests were performed comparing * particle localization and localization $\%$ change to that of PS. A p-value $<0.05$ was considered statistically significant.

Fig. 4 Adhesion of sLe ${ }^{\text {a }}$-coated Si and Ti spheres (500 nm ESD) to inflamed endothelium after 5 min of particle-loaded RBC flow $\left(5 \times 10^{5}\right.$ particles $/ \mathrm{mL}$ blood $)$ at various shear rates. Experiments were done with the endothelial layer at the bottom of the chamber. Particle binding is reported as the number of adherent particles per area. Cell normalized binding is reported as the number of adherent particles per endothelial cell (EC). Student t-tests were performed comparing * $\mathrm{Si}$ and $\mathrm{Ti}$ adhesion at a given shear rate. A p-value $<0.05$ was considered statistically significant.

Fig. 5 Adhesion of sLe ${ }^{\mathrm{a}}$-coated PS, Si, and Ti spheres (500 nm ESD) to inflamed endothelium after 5 minutes of particle-loaded RBC flow $\left(5 \times 10^{5}\right.$ particles $/ \mathrm{mL}$ blood $)$ at $200 \mathrm{~s}$ ${ }^{1}$. Experiments were done in a recirculation flow PPFC setup with the endothelial layer at the bottom $(A, B)$ or top $(C, D)$ of the chamber. Particle binding is reported as the number of adherent particles per area. Cell normalized binding is reported as the number of adherent particles per endothelial cell (EC). Student t-tests were performed comparing particle adhesion to $* \mathrm{PS}$ and ${ }^{\#} \mathrm{Si}$ at the same chamber orientation. A p-value $<0.05$ was considered statistically significant.

Fig. 6 Adhesion of sLe ${ }^{\mathrm{a}}$-coated PS, Si, and Ti spheres (500 nm ESD) to inflamed endothelium after 5 minutes of particle-loaded RBC flow $\left(5 \times 10^{5}\right.$ particles $/ \mathrm{mL}$ blood $)$ at $200 \mathrm{~s}$ 1 . Experiments were done in a recirculation flow PPFC setup with the endothelial layer at the bottom $(A, B)$ or top $(C, D)$ of the chamber. Particle binding is reported as the number of adherent particles per area. Cell normalized binding is reported as the number of adherent particles per endothelial cell (EC). Student t-tests were performed comparing particle adhesion to $* \mathrm{PS}$ and ${ }^{\#} \mathrm{Si}$ at the same chamber orientation. A p-value $<0.05$ was considered statistically significant. 


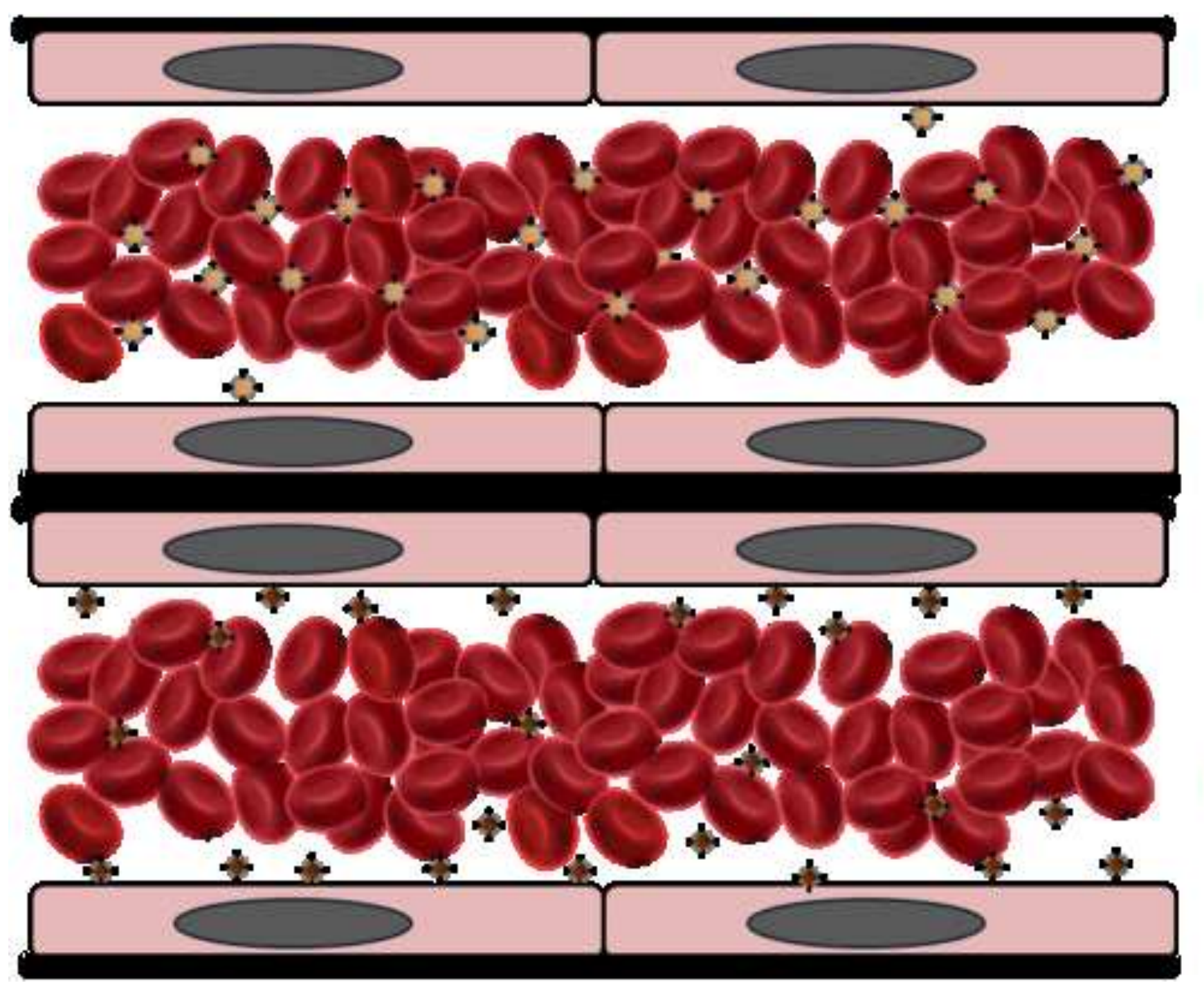

- Nanosphere $\rho_{N S}=\rho_{\text {Blood }}$

\section{Red Blood Cell}
* Nanosphere
$\rho_{N S} \geqslant \rho_{B l o o d}$
Red Blood Cell
* Nanosphere
$\rho_{N S} \geqslant \rho_{B l o o d}$
Red Blood Cell
* Nanosphere
$\rho_{N S} \geqslant \rho_{B l o o d}$
Red Blood Cell

.




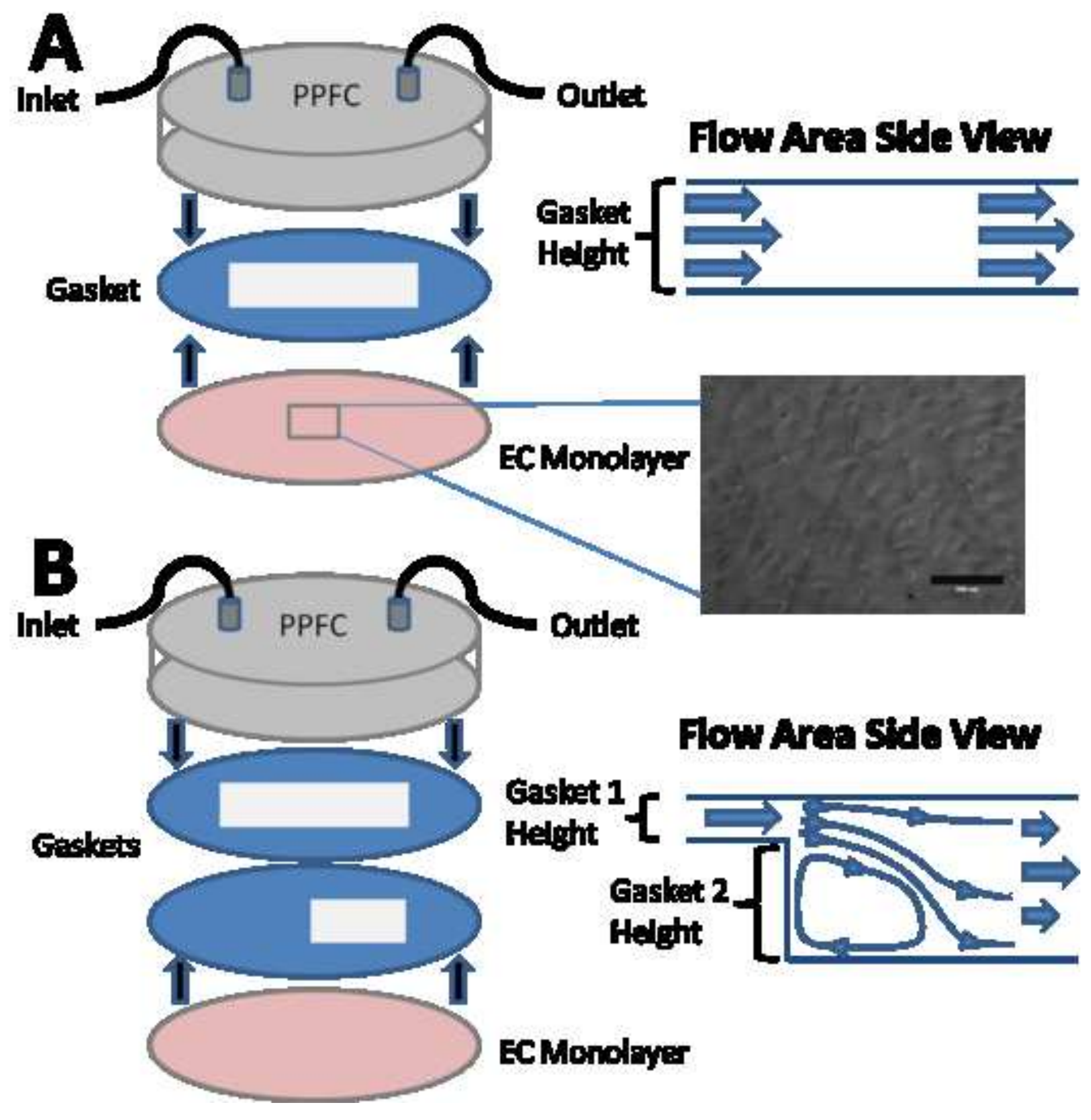



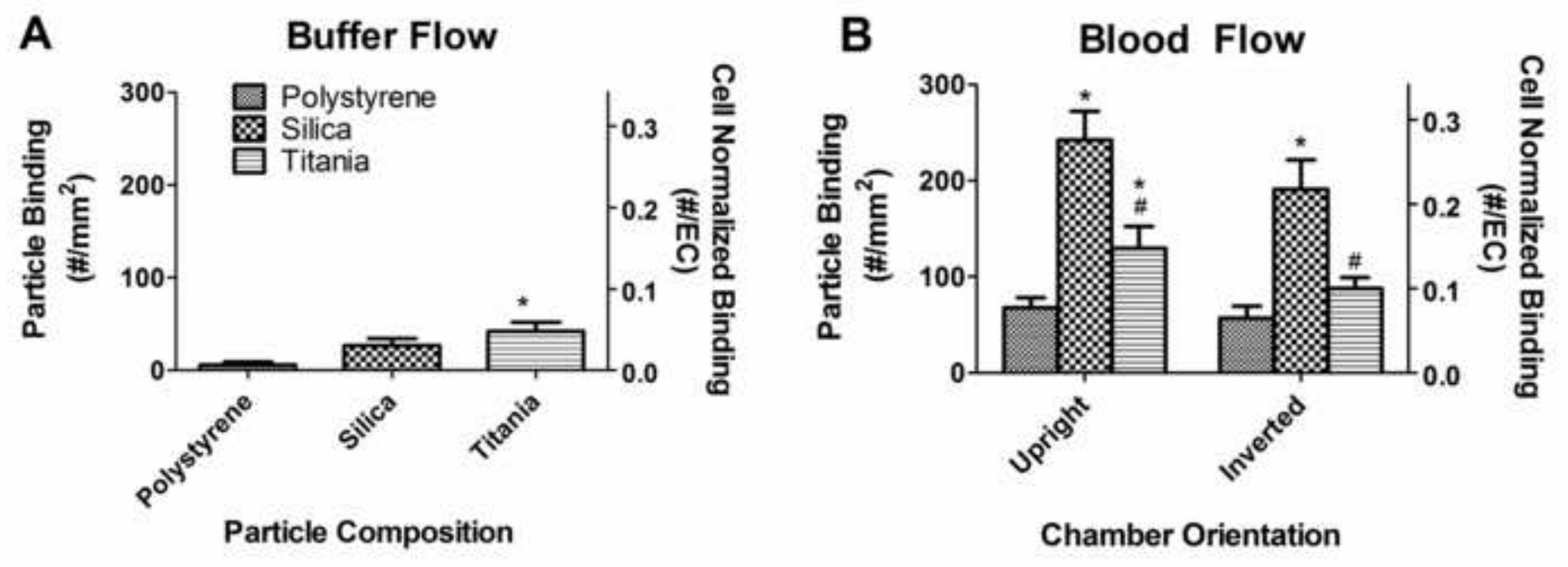


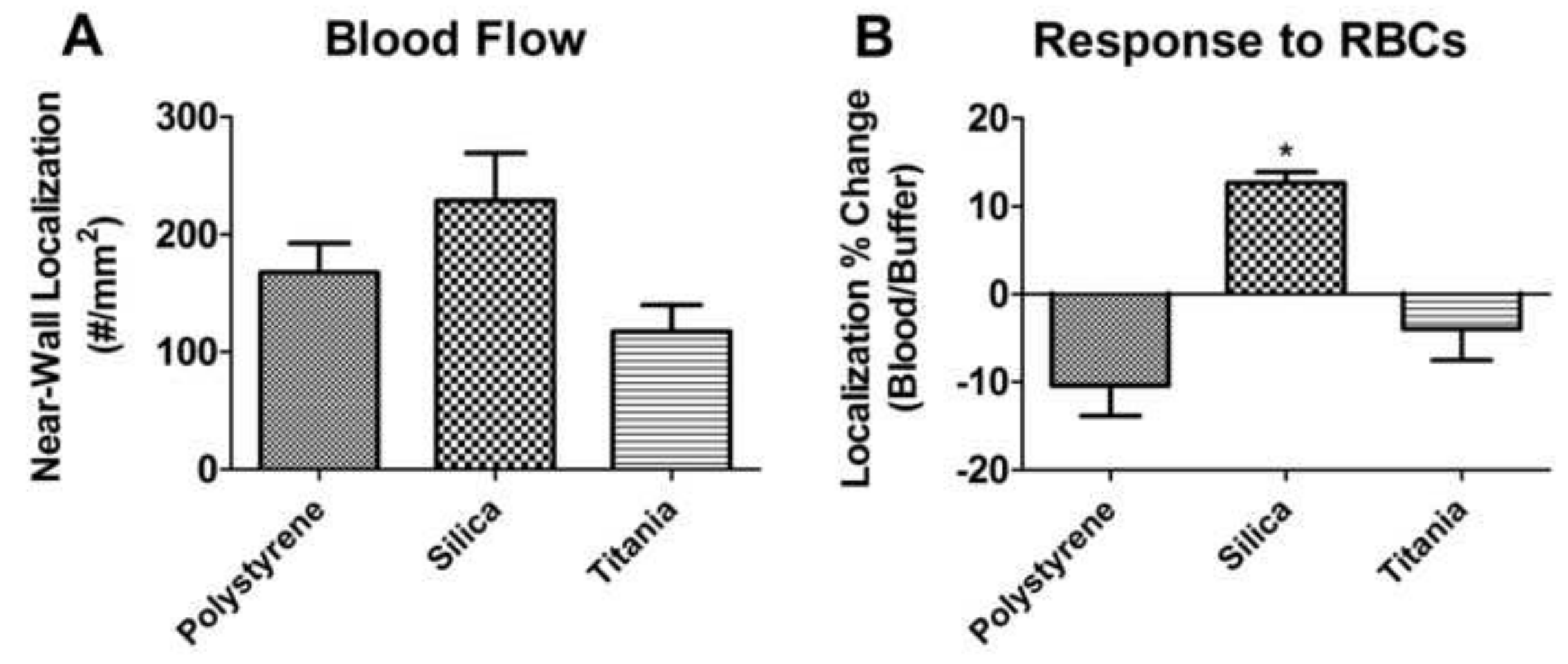




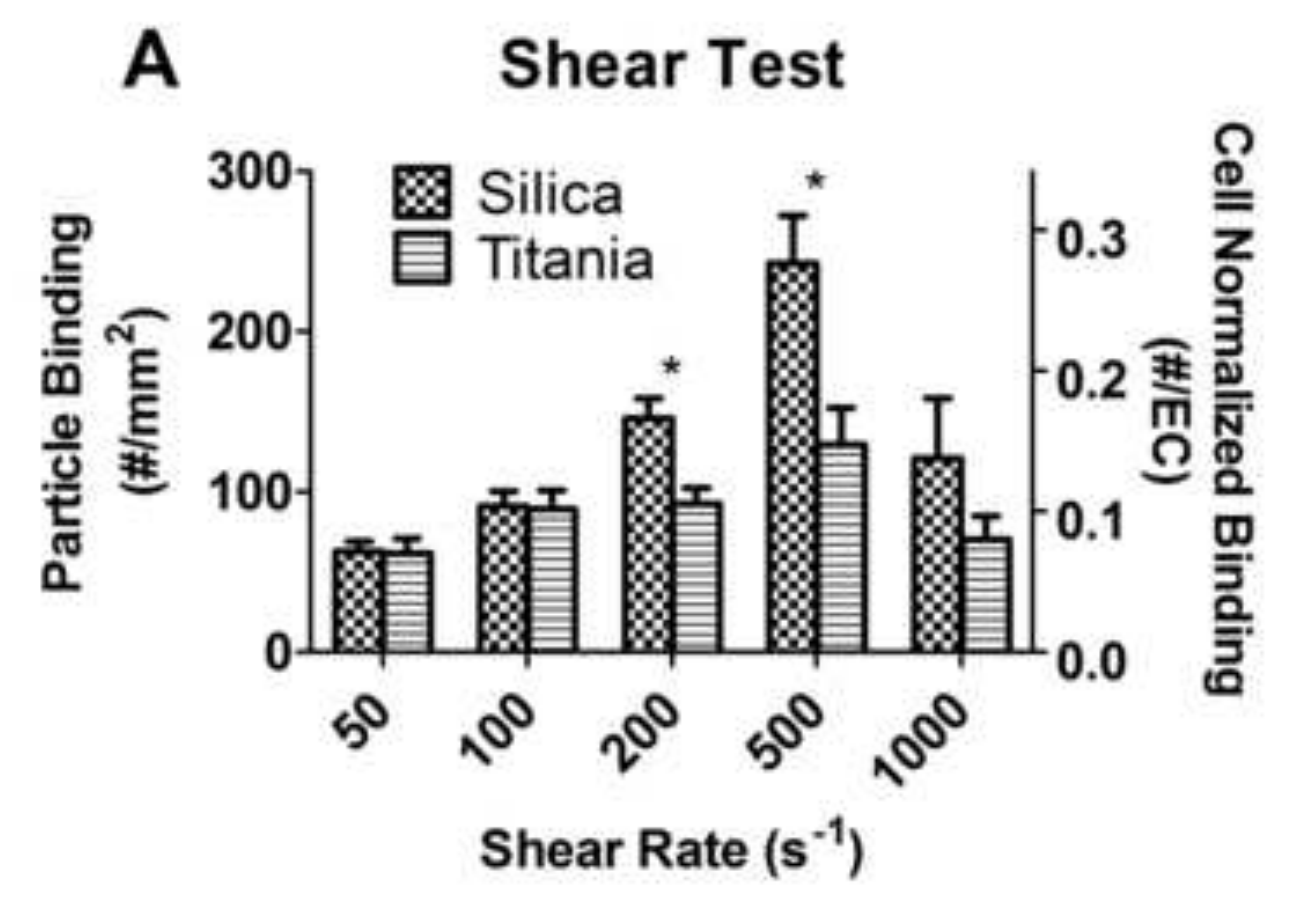

B Normalized Shear Test

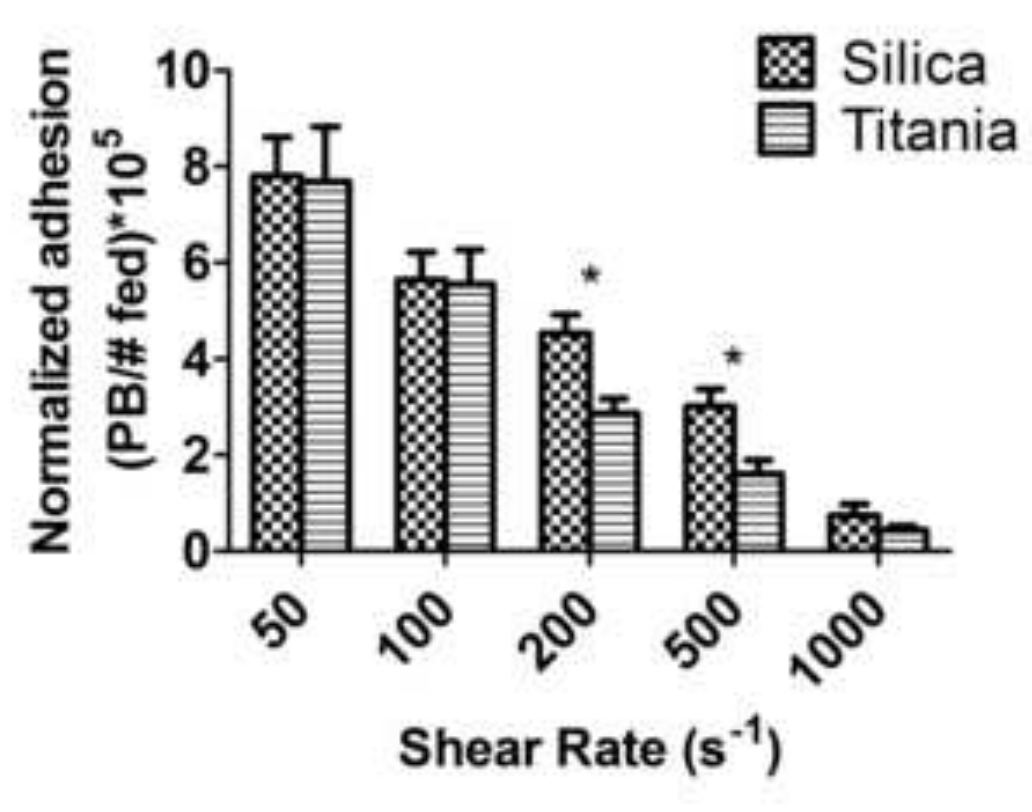




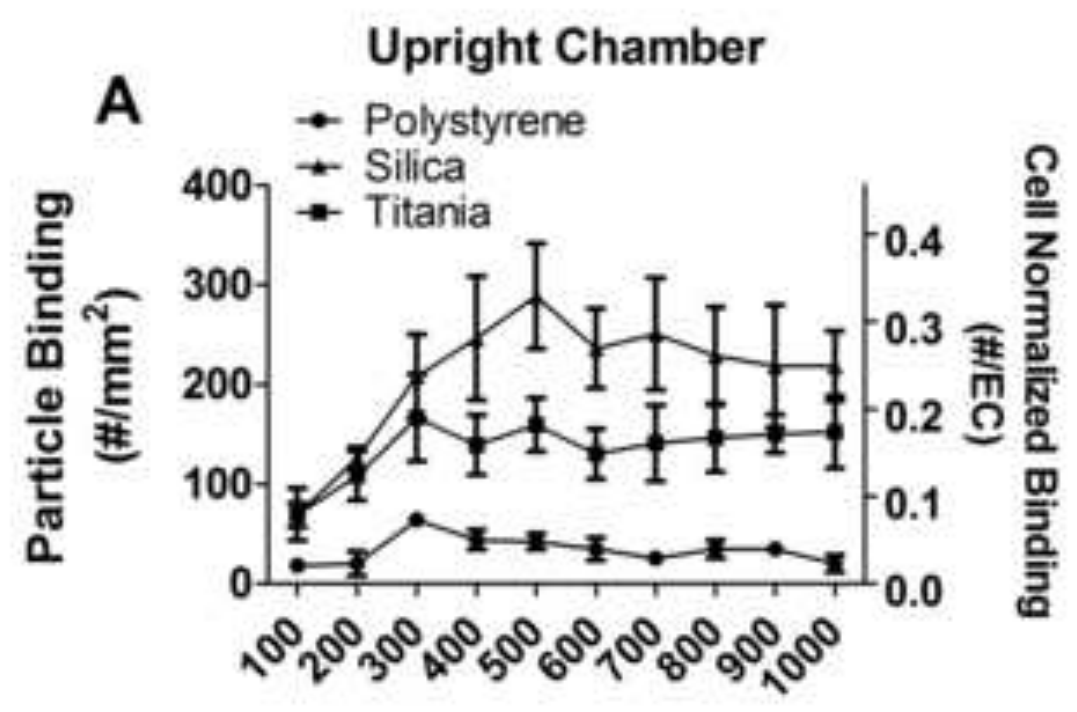

Distance From Step $(\mu \mathrm{m})$

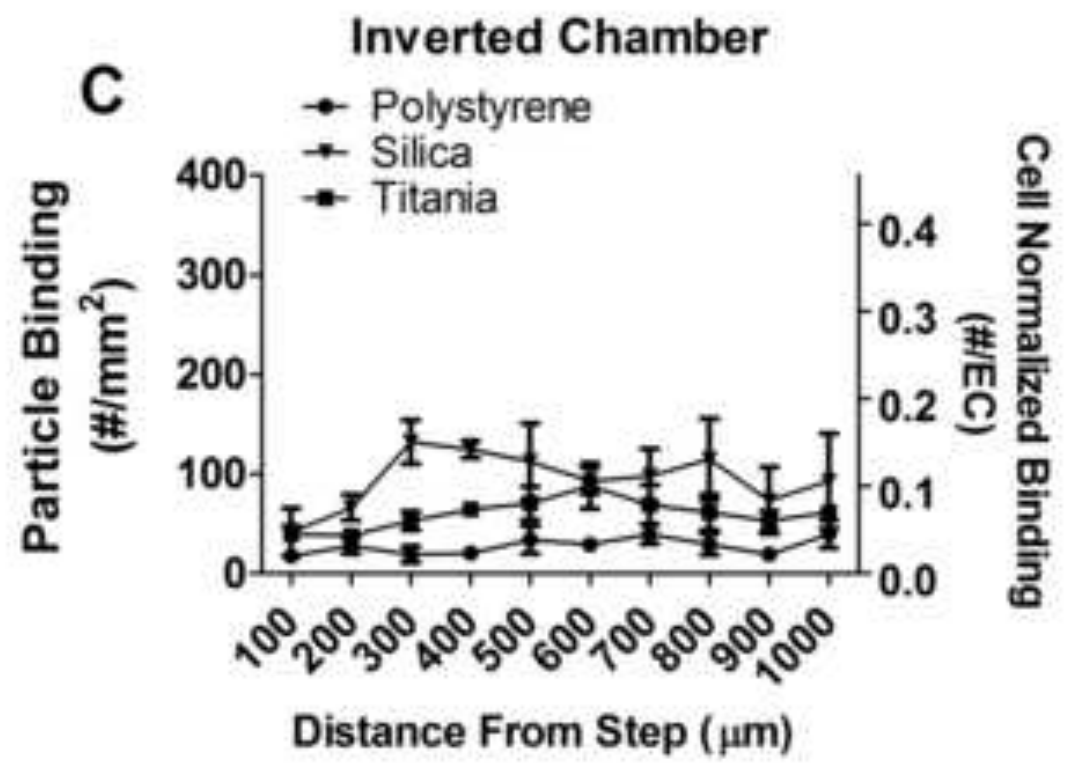

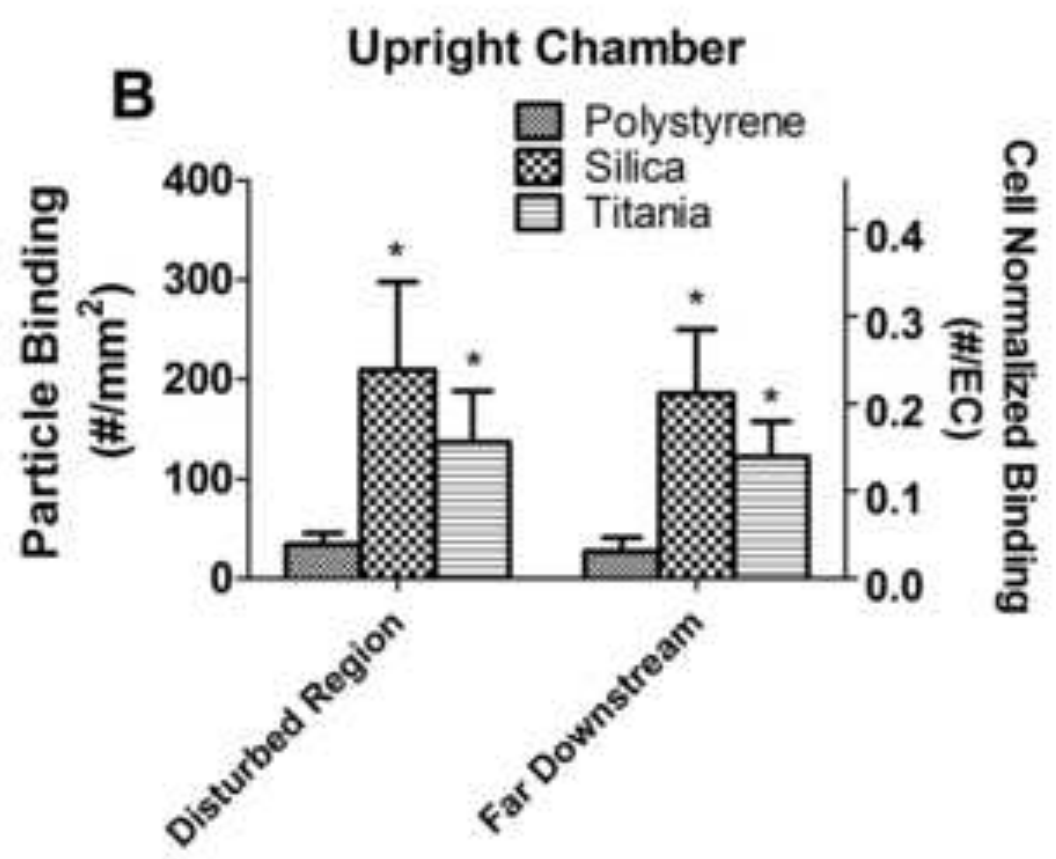

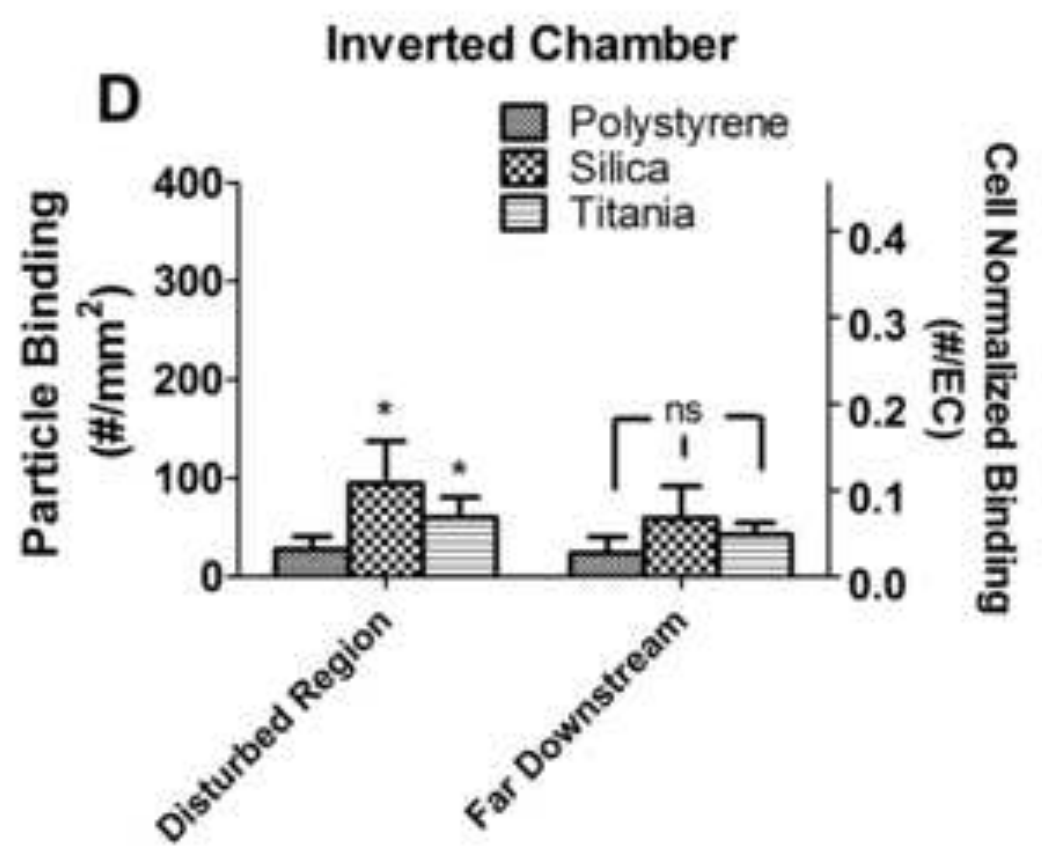



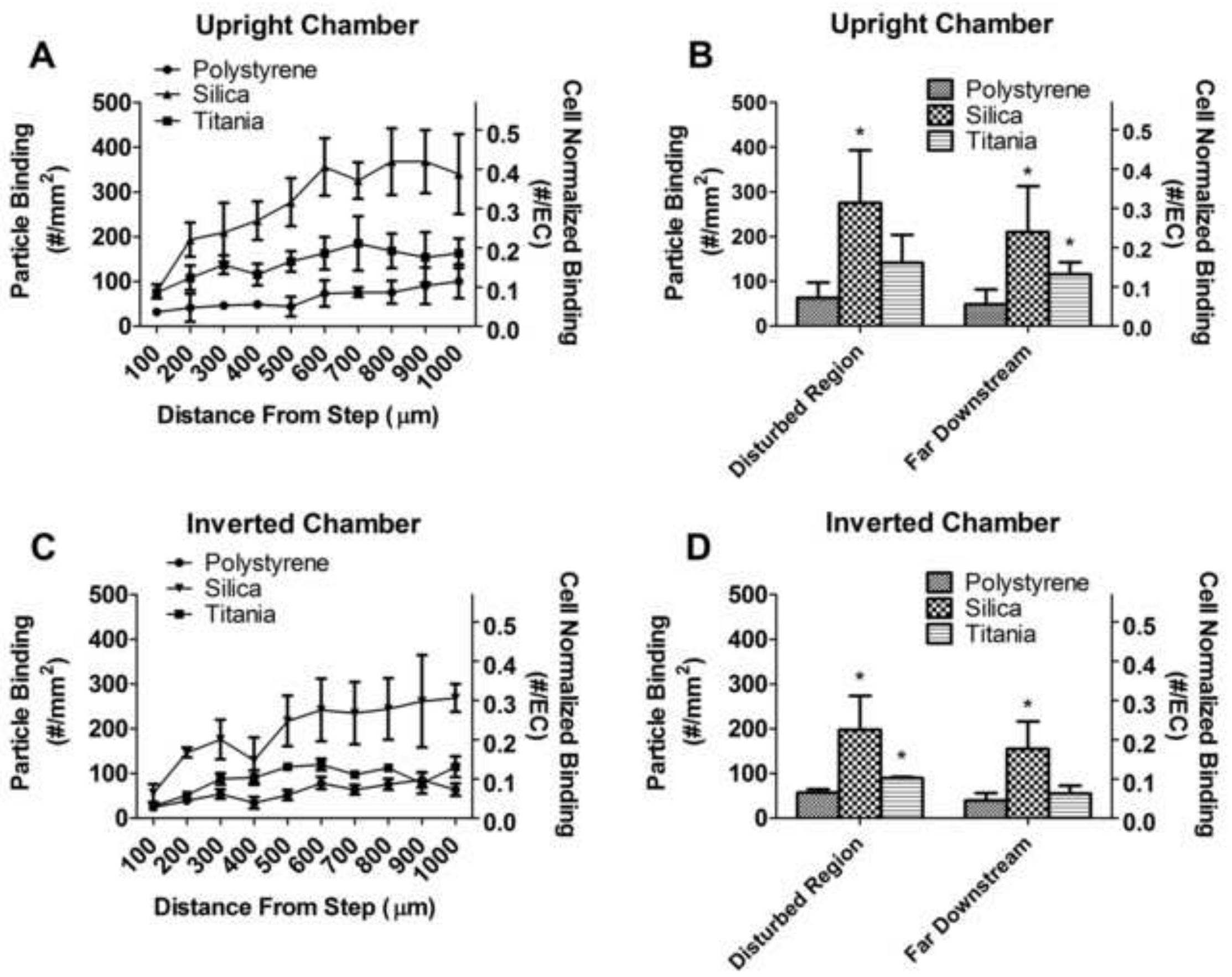